\title{
La ruleta climática en Bolivia: Vulnerabilidad ante la incertidumbre y sus efectos
}

\author{
Climate roulette in Bolivia: Vulnerability to uncertainty and its \\ effects on water supply
}

\author{
Olivera-Villarroel, S.; Candia-Calderon, A. G.; Borja-Vega, Ch.; Editor \\ academico Prof. Doctorante Edgar Marinero Orantes
}

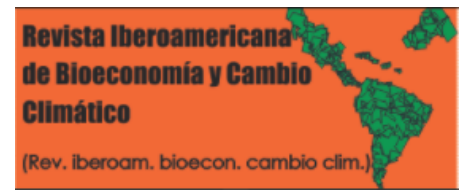

\author{
D. Olivera-Villarroel \\ satzcha@msn.com \\ Universidad Autónoma Metropolitana de México, \\ Mexico \\ (iD A. G. Candia-Calderon \\ gabyalethea@gmail.com \\ Universidad Autónoma Metropolitana de México, \\ Mexico \\ iD Ch. Borja-Vega \\ cborjavega@worldbank.org \\ Banco Mundial, United States \\ Editor academico Prof. Doctorante Edgar Marinero \\ Orantes \\ edgar.marinero@ues.edu.sv \\ Universidad de El Salvador, El Salvador
}

Revista Iberoamericana de Bioeconomía y Cambio

Climático

Universidad Nacional Autónoma de Nicaragua, León, Nicaragua ISSN-e: 2410-7980

Periodicidad: Semestral

vol. 7, núm. 14, 2021

czuniga@ct.unanleon.edu.ni

Recepción: 08 Septiembre 2021

Aprobación: 18 Noviembre 2021

URL: http://portal.amelica.org/ameli/journal/394/3941761009/

DOI: https://doi.org/10.5377/ribcc.v7i14.12810
Resumen: Bolivia es uno de los países con mayor disponibilidad de agua en el mundo; sin embargo, posee un déficit anual de agua que afecta al $13 \%$ de la población urbana y al $38 \%$ de la población rural; además, es el país con menor capacidad de almacenamiento en embalses de agua de la región. Por lo que la presente investigación analiza los efectos del cambio climático en el sector agua y sus impactos en la vulnerabilidad de las áreas urbanas del país. Para el efecto, se utilizó el índice de vulnerabilidad al cambio climático (IVCC), que aplica el método de descomposición de Shapley que identifica los pesos ponderados de los componentes del IVCC. Entre los hallazgos se destaca que el principal cambio por el que atravesará Bolivia será el aumento a la exposición de las sequías, por lo que es de vital importancia diversificar las fuentes de agua; además, dado el aumento poblacional en las ciudades capitales se debe mejorar la distribución de agua potable y el sistema de alcantarillado. En relación con las ciudades pequeñas se deben generar mecanismos de riego y saneamiento para solventar los posibles impactos climáticos futuros. Finalmente, se debe generar una cultura de manejo del agua -re uso de aguas residuales, uso moderado del agua potable, entre otros-. Es de vital importancia priorizar estas políticas públicas dado que el acceso al agua potable y saneamiento es un derecho humano el cual se debe garantizar para el desarrollo social y económico de Bolivia.

Palabras clave: Índice de vulnerabilidad al cambio climático, Agua potable, Cambio climático, Bolivia, Shapley.

Abstract: Bolivia is one of the countries with the highest availability of water in the world; however, it has an annual water deficit that affects $13 \%$ of the urban population and $38 \%$ of the rural population; Furthermore, it is the country with the lowest storage capacities in water reservoirs in the region. The research analyzes the effects of climate change in the water sector and its impacts on the vulnerability of urban areas of the country. For this purpose, the climate change vulnerability index (CCVI) was used, which applies the Shapley decomposition method that identifies the weighted weights of the CCVI components. Among the findings, it is highlighted that the main change that Bolivia will go through will be the increase in exposure to droughts, which is why it is vitally essential to diversify water sources; Furthermore, given the population increase in the capital cities, the distribution of drinking water and the sewerage 
system must be improved. Concerning small cities, irrigation and sanitation mechanisms must generate to solve future climate changes. Finally, a water management culture must be generated - reuse of wastewater, moderate use of drinking water, among others. It is vitally important to prioritize these public policies, given that access to drinking water and sanitation is a human right that must be guaranteed for the social and economic development of Bolivia.

Keywords: Index of vulnerability to climate change, Drinking water, Climate change, Bolivia, Shapley.

\section{INTRODUCCIÓN}

Bolivia se encuentra en el centro de Sudamérica, dada su ubicación geográfica posee zonas diversas: comenzando con mesetas altas que alcanzan más de 4.000 metros sobre el nivel del mar, pasando por valles de elevación media y terminando en planicies tropicales rodeadas de parte de la Amazonía y la Chiquitanía y en el sur concluye con la región del Chaco (chaco boreal y parte del pantanal). Como resultado de esta variedad existe una amplia gama de temperaturas y microclimas dentro del país.

Sin embargo, esta diversidad se ve afectada por el cambio en la dinámica climática que ha llevado a impactos adversos acelerados, el más notorio siendo la pérdida de aproximadamente el $50 \%$ de la superficie de los glaciares existentes en la región cordillerana de Bolivia, por lo que se esperan temperaturas más altas y mayor intensidad en las precipitaciones durante la temporada de lluvias, que expondrá las diferentes regiones del país a períodos secos prolongados y a un aumento en la frecuencia y magnitud de las inundaciones, tormentas de granizo, ríos desbordados, deslizamientos de tierra, heladas, entre otros. (Banco Mundial, 2013, 2019; Rangecroft et al., 2015; WRI, 2017).

Evidencia de lo anterior son: los fenómenos del “El Niño” en 2007 y “La Niña” en 2008, que ocasionaron fuertes lluvias que causaron inundaciones y deslizamientos de tierra e impactaron a más de 258,000 personas. El impacto económico de estos fenómenos climáticos se estimó en alrededor de $\$ 443$ millones, casi el 4 por ciento del PIB de 2007, con pérdidas principalmente en infraestructura dañada, en particular carreteras y producción agrícola, incluyendo activos de los hogares más pobres y vulnerables. (McKenzie 2005; Banco Mundial. 2013.) Las fuertes lluvias estacionales en 2013 y 2014 causaron graves inundaciones y deslizamientos de tierra, que afectaron a más de 44,000 hogares en 113 municipios (DESINVENTAR, 2018). El último evento en el otoño de 2019, el devastador incendio de más de 4.5 millones de hectáreas en las zonas de: la Amazonía, el Chaco y la Chiquitania, este incendio se extendió por más de 3 meses y es considerado uno de los mayores daños ambientales del mundo en las últimas décadas (Fundación Amigos de la Naturaleza, 2019).

Los desastres ambientales mencionados anteriormente conllevan a pensar que Bolivia se encamina a un clima más cálido y seco, razón por la cual se tiene que identificar cómo se encuentra el sector de agua y saneamiento en el país, dado que este sector es uno de los más afectados por el cambio climático (Sanders et al, 2019). El CAF, en su informe "Agua y saneamiento en el Estado Plurinacional de Bolivia" menciona que: "(dado que ...) la variabilidad espacial y temporal es elevada, por lo que se requiere el almacenamiento y regulación de excedentes hídricos. Bolivia solo cuenta con una capacidad de almacenamiento en embalses de unos 56 por habitante, cuando Ecuador y Perú, con geografías y climas equivalentes, disponen de 489 y 190 , respectivamente" (p.22).

Como consecuencia de la baja capacidad del sector de agua potable y saneamiento el país está sujeto a un incremento progresivo de la vulnerabilidad ante el cambio climático y manifiestan el bajo nivel de resiliencia del país. Es en este sentido que, la presente investigación busca, por un lado, identificar los niveles 
de vulnerabilidad que poseen los municipios urbanos del país ${ }^{[1]} \mathrm{y}$, por el otro, -considerando el aumento de las sequías y la importancia que tiene el acceso al agua potable para el desarrollo social y económico- busca analizar los efectos del cambio climático en este sector y sus impactos en el aumento de la vulnerabilidad ambiental. Ambos objetivos sirven para generar evidencia que contribuya a la reorientación de las políticas públicas sectoriales para abatir los efectos del cambio climático.

El presente artículo se divide en 6 partes: en el primer apartado, se desarrollan las características metodológicas del estudio, donde se muestra la forma de construcción del índice de vulnerabilidad, el subíndice del sector agua y la descomposición de Shapley (Israeli 2007). En el segundo apartado, se da a conocer los principales resultados del índice de vulnerabilidad al cambio climático. Posteriormente, en el tercer apartado, se realizó un subíndice del sector agua y se dio a conocer los principales municipios vulnerables en este sector. El cuarto apartado muestra la construcción del índice de principales amenazas del cambio climático y con la ayuda del modelo climático MIROC 5.0 y MPI 5.0 RCP 4.5 ${ }^{[2]}$, se efectúa una comparación de una línea base 2004 a 2014 versus el comportamiento de las variables climáticas futuras entre 2066 a 2076. Con base en los resultados recabados se efectuó un breve listado, en el apartado 5, de posibles políticas públicas que ayuden a mitigar los efectos del cambio climático en los municipios analizados. Finalmente, se muestran las principales conclusiones en el apartado 6. Ver anexos 1, 2, 3, y 4

\section{Materiales y metodología}

\section{1. Índice ponderado de Vulnerabilidad al Cambio Climático}

Para el desarrollo de la investigación se utilizó el índice ponderado de Vulnerabilidad al Cambio Climático (IVCC), diseñado por el Banco Mundial para Bolivia en 2019 (Sanders, K; Miranda, J y Olivera, S., 2019). Debido a que el 62\% de la población boliviana se encuentra en los municipios de más de 10.000 habitantes, y dados los riesgos latentes que pueden incidir en un mayor número de personas en estos municipios, se optó por tomar como unidad de análisis 59 municipios que cumplen con las características poblacionales antes mencionadas.

Es importante mencionar que al no incorporase en el estudio los municipios rurales no se capta la vulnerabilidad total del país, dado que estos municipios en su mayoría no cuentan con accesos a servicios básicos. Sin embargo, las zonas urbanas tienen un mayor potencial de acumular impactos negativos del cambio climático debido a las conglomeraciones poblacionales y de activos (IPCC 2001). Por ende, la priorización para mitigar estos efectos en las ciudades se torna imperante en la creación de política pública (Bai et al., 2018).

Tal como se observa en el diagrama 1, las principales dimensiones que se utilizaron en el índice fueron: 


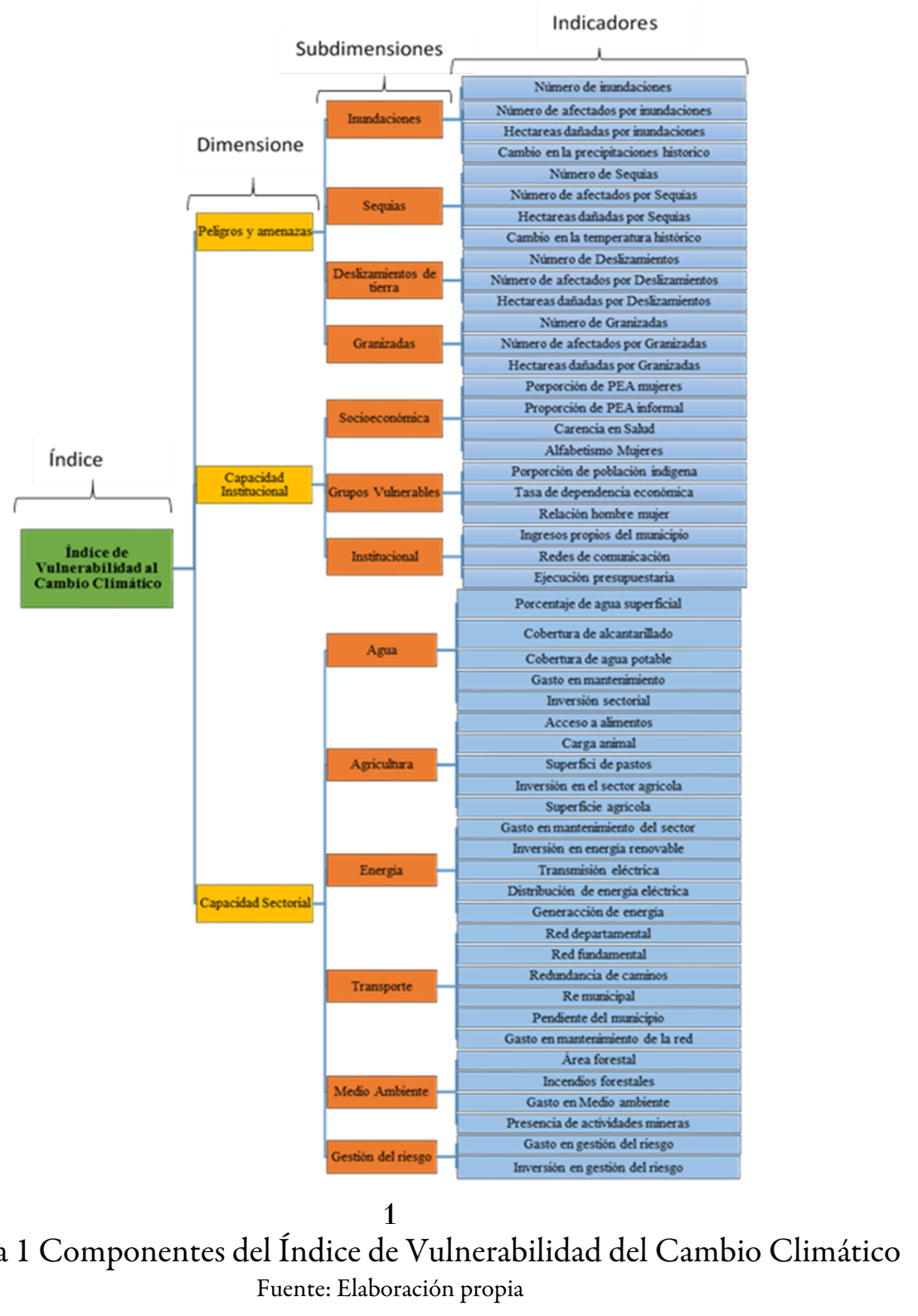

1) Peligro y amenazas, integra la exposición y la vulnerabilidad física. Captura eventos que podrían ocurrir y la población que podría estar potencialmente expuesta a estos eventos (Mcguire 2007). Las principales fuentes de estudio para esta dimensión fueron las bases de datos del Sistema de inventario de efectos de desastres (DESINVENTAR) 1980 - 2015 y las bases del Sistema de alerta temprana de MIROC 3.0 - 5.0 CIIFEN -CHRIPS 1980-2000 / 2000 a 2010, estas bases brindan información sobre el número de desastres, los cambios en la distribución general de precipitación y temperatura mes a mes en los años de análisis del índice y daños agregados de población afectada y superficie.

2) Capacidad institucional se centra en la susceptibilidad de las comunidades y centros urbanos a esos peligros. Capta la fragilidad para hacer frente a una situación de crisis derivada de las contingencias climáticas de los sistemas institucionales, socioeconómicos y poblacionales. Todos los datos sociodemográficos se obtienen del censo de Población y Vivienda 2012, información del Índice de Riesgo Municipal desarrollada por el Programa Mundial de Alimentos e informes del Viceministerio de Economía y la Unidad de Análisis de Políticas Sociales y Económicas (UDAPE). 
3) Capacidad sectorial abarca la resiliencia de la infraestructura que cuenta el país para hacer frente a una crisis ambiental. Las fuentes de información de los datos fueron: GEOdata, del ministerio de Defensa de Bolivia; la red de información geográfica, del Instituto Nacional de Estadística (INE); así como las bases de datos de la Unidad de Análisis de Políticas Sociales y Económicas (UDAPE). El índice tiene un rango entre cero a uno, el valor cero significa que el municipio tiene el nivel más bajo de riesgo de desastre y el valor uno implica lo contrario, es decir que el municipio está situado en el nivel más alto de riesgo de desastres (Beccari 2016).

El IVCC es ponderado por categorías donde el peso de cada componente se calculó a partir de la descomposición de Shapley que estima las contribuciones relativas de las diversas variables explicativas y la contribución a la varianza de cada componente particular del índice (Nguefack-Tsague et al 2011). Esto hace que el índice sea consistente ante las variaciones de los componentes y explique, a su vez, los niveles de relevancia de las variables incorporadas (Barnett et al, 2008), ver diagrama 2.

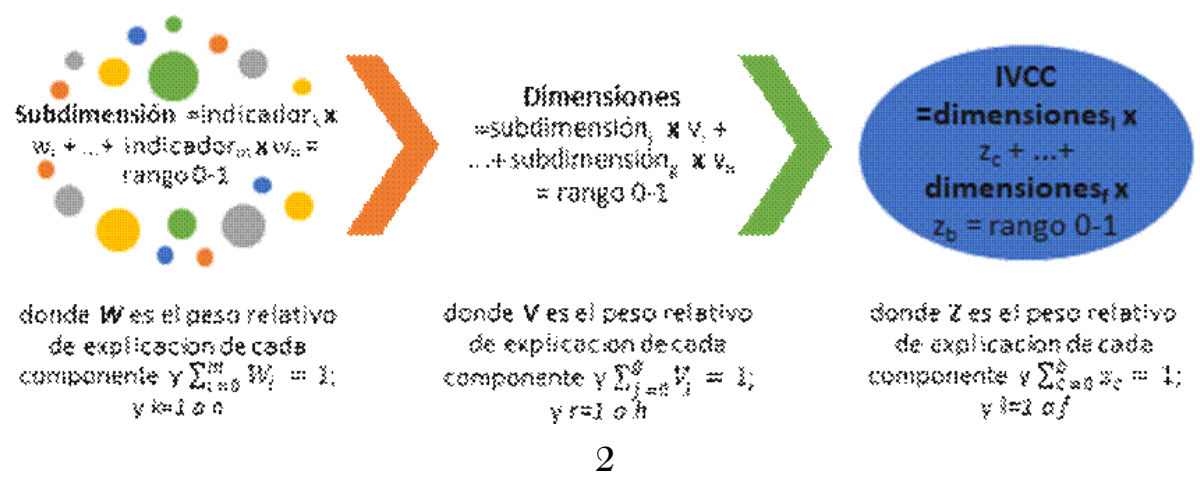

Diagrama 2: Construcción del Índice de Vulnerabilidad al Cambio Climático Fuente: elaboración propia

Los pasos para la construcción del método son:

1) Se genera un índice con ponderaciones promedios o iguales.ec 1

$$
\text { IVCC }=\frac{1}{3} * \text { peligroyamenazas }_{+} \frac{1}{3} * \operatorname{sensibilidad~}_{+} \frac{1}{3} * \text { capacidad }_{-}
$$

2) En una segunda etapa se calcula el porcentaje de contribuciones de las variables que componen el índice a la varianza total del índice previamente estimado. ec 2

$$
\theta=\beta_{1} * x_{1}+\beta_{2} * x_{2}+\cdots+\beta_{k}^{*} x \square_{k}
$$

Donde es el índice, representa la contribución relativa de cada componente usado al índice, que en un primer paso tiene igual valor $\beta_{-} \mathrm{i}=\beta_{-} \mathrm{j}$ y deben sumar 1 para mantener la identidad y congruencia en la estimación de la descomposición a ser desarrollada (Shorrocks, 1999) y x1, x2,..., xk representan los componentes del índice, entonces el cuadrado $\mathrm{R}$ parcial para la variable xj puede ser calculado por la descomposición de Shapley-Owen (Zaiontz, 2017), ec 3

$$
R=\sum_{\mathrm{TCV}-\left(X_{j}\right)}\left(\frac{R^{2}\left(\mathrm{TU}_{(\mathrm{xj})}\right)-R^{2}(T)}{\mathrm{k} * \mathrm{C}(k,-, 1,|T|)}\right)
$$


Donde $\mathrm{V}=\{\mathrm{x} 1, \mathrm{x} 2, \ldots, \mathrm{xk}\} \mathrm{y}|\mathrm{T}|=$ el número de elementos en algún subconjunto $\mathrm{T}$ de $\mathrm{V}$. También $\mathrm{R}$ cuadrado $(T)=$ el valor de $R$ cuadrado para la regresión de los componentes en $T$ en $\Theta$, que tenderá a valores cercanos a 1 en el caso de aplicarse a la ponderación de índices (OECD, 2008). Suponemos que R cuadrado $(\varnothing)=0$.

Con el método anterior se logra calcular el peso de cada subcomponente, de esta manera cada categoría se evalúa del cero al uno y al ser agregado a la siguiente dimensión y ponderarse respecto a la representatividad relativa de sus componentes (Becker et al, 2017), se puede volver a leer del cero al uno. Donde los niveles cercanos al 0 indican menor vulnerabilidad y municipios con niveles cercanos al 1 indican mayor vulnerabilidad. El índice se acota a 1 para poder identificar la composición óptima de variables que explican lo más cercano a la totalidad de la variación en vulnerabilidad.

\section{2. Índice de temperatura y precipitaciones en Bolivia}

Para analizar los impactos del cambio climático se generó un índice de tendencia de temperatura y precipitación en Bolivia con la finalidad de evaluar la variabilidad y volatilidad mensual de estas variables climáticas en comparación a una línea base de 2004 - 2014 en comparación con el comportamiento de las variables climáticas futura entre 2066 a 2076, usando para ello información generada por los modelos climáticos MIROC 5.0 y MPI 5.0 bajo el escenario RCP 4.5. Debido a la gran diversidad de climas y condiciones atmosféricas observables en el territorio boliviano se considera que un aumento o disminución de las variables evaluadas es igualmente perjudicial (IMCO, 2012).

La medición de la distancia de cada punto, que representa un municipio, al origen delimita el área de influencia, y con ello dio lugar a estimar un indicador desagregado de la vulnerabilidad climática, que arroja información por cada mes del año. Donde (xi, yi) son las coordenadas que denotan el cambio porcentual en variabilidad y nivel respectivamente. Las coordenadas con las que se quiere comparar son $(0,0)$, entonces la fórmula final se expresa de la siguiente forma ecuación 4 :

$$
d=\sqrt{ }\left(x^{2}+y^{2}\right)
$$

o El indicador tiene ciertas características deseables:

Es útil para comparar todas las áreas urbanas. Información sobre el cambio de nivel y variabilidad del clima. Presenta un valor óptimo $(\mathrm{d}=0)$ que sirve como parámetro para evaluar el desempeño del clima en las regiones. El método es replicable a partir de datos simples, ya sean binarios o categóricos en la variable dependiente.

o El método utilizado tiene una limitación importante:

Es incapaz de distinguir tendencias en los cambios de nivel del clima. El modelo no permite conocer si un municipio es más cálido o frío, seco o lluvioso en comparación con años anteriores.

Para dar solución a este problema fue necesario crear etiquetas que clasificaran a los municipios de acuerdo con las características climatológicas cualitativas que los diferencian del resto de las ciudades, ver tabla 1 y 2. Las etiquetas se definen de la siguiente manera.

TABLA 1.

Criterios de definición de tendencias de Temperatura para áreas urbanas.

\begin{tabular}{ll}
\hline Tipos de Area & Tendencia \\
\hline Areas urbanas cálidas & si la localidad ha presentado un ammento de la temperatura en más de 6 \\
& meses a año. \\
Areas urbanas frias & si la temperatura ha de scendido a to largo del tiempo en más de 6 meses \\
& al año. \\
\hline
\end{tabular}


Fuente: elaboración propia

TABLA 2

Criterios de definición de tendencias de precipitación para áreas urbanas.

\begin{tabular}{ll}
\hline Tipos de Area & Tendencia \\
\hline Areas urbanas lluviosas & $\begin{array}{l}\text { Si la región ha experimentado nivel es superiores đe lfmía ent al metos } 6 \\
\text { meses đel año en comparación a años pasados. }\end{array}$ \\
Areas urbanas secas & $\begin{array}{l}\text { Si la tendencia de precipitación es a la baja en comparación a décadas } \\
\text { anteriores. }\end{array}$ \\
\hline
\end{tabular}

Es importante contar con estos indicadores (Adger et al, 2004) para poder clasificar a los municipios de acuerdo con su tendencia de vulnerabilidad en temperatura y precipitación que han seguido en las tres décadas bajo el análisis. Este indicador mide los cambios en la volatilidad para cada mes del año. Presenta un valor óptimo que sirve como parámetro para evaluar el desempeño del clima en las regiones. Es decir, mientras más pequeño el índice de tendencia de volatilidad, menor la volatilidad de la variable analizada en un área urbana en particular

En forma similar en la tabla 3, es posible saber qué tan volátil o estable es un municipio con las siguientes etiquetas

TABLA 3.

Criterios de definición de volatilidad climática en los municipios del estudio.

\begin{tabular}{ll}
\hline Tipos de Área & Tendencia \\
\hline tocalidad estable & si la variable evaluada está consolidando sn volatilidad en màs de 6 \\
& meses. \\
localidad volátil & si en más de 6 meses del ào el área urbana ha experimentado aumentos \\
& en la variabilidad del clima. \\
\hline
\end{tabular}

Fuente: elaboración propia

\section{Resultados y Discusiones}

\subsection{Cambio climático}

Con base en el índice de tendencia de temperatura y precipitación en Bolivia se destaca que, en comparación a la línea base los escenarios futuros muestran -en términos generales- que, el país tiene tendencias a un clima más seco y cálido, aunque con prevalencias a eventos de precipitación extremos en los meses de verano oscilantes. Además, tal como se observa en el gráfico 1, las condiciones climáticas del país presentan tendencias futuras de un clima más cálido y seco, lo que incrementa las probabilidades de escenarios de sequías a nivel general a lo largo de Bolivia.

Si bien esta tendencia de clima seco y cálido disminuye las condiciones generales para el acontecimiento de inundaciones y deslizamientos de tierra, en términos generales, las probabilidades de este tipo de eventos extremos no disminuyen sustancialmente y de hecho se mantiene los escenarios de inundaciones en regiones como el área metropolitana de Cochabamba y los municipios de la cuenca amazónica (Ishizawa et al., 2017). Sequías y granizadas en municipios como La Paz y Tarija demuestran dicha variabilidad climática. Inundaciones en Sucre, Tarija y los municipios de la región amazónica y deslizamientos de tierra en el Alto, La Paz y Sucre también generaron importantes pérdidas económicas (Ishizawa et al., 2017), Grafico 1. 


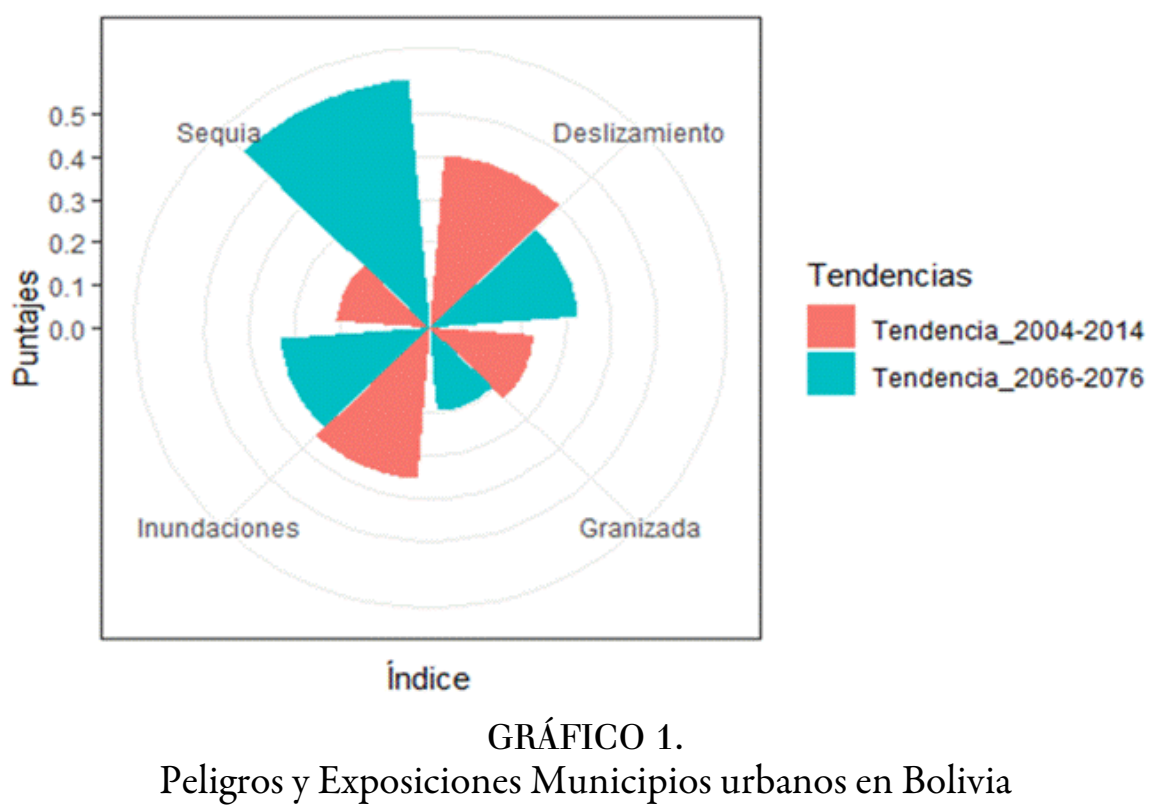

Fuente: elaboración propia usando los modelos climáticos MIROC 5.0 y MPI 5.0 RCP 4.5. Nota: municipios urbanos de Bolivia. El rango de medidas del índice va del cero al uno, donde cero es un índice de menor peligro y uno una posición de peligro muy elevado

Por otro lado, si analizamos la distribución de amenazas climáticas tanto históricas como las de escenarios de cambio climático, se identifica un cambio en la distribución entre las ciudades grandes, tanto en extremos como en la media de la distribución; mientras que en las ciudades medianas y pequeñas hay un cambio en la media de la distribución, pero no en los extremos de amenazas en la distribución, grafico 2 . Esto evidencia el incremento en las amenazas de las ciudades grandes debido en gran medida a la cantidad de población existente en los centros urbanos como al incremento en la probabilidad de eventos climáticos extremos.

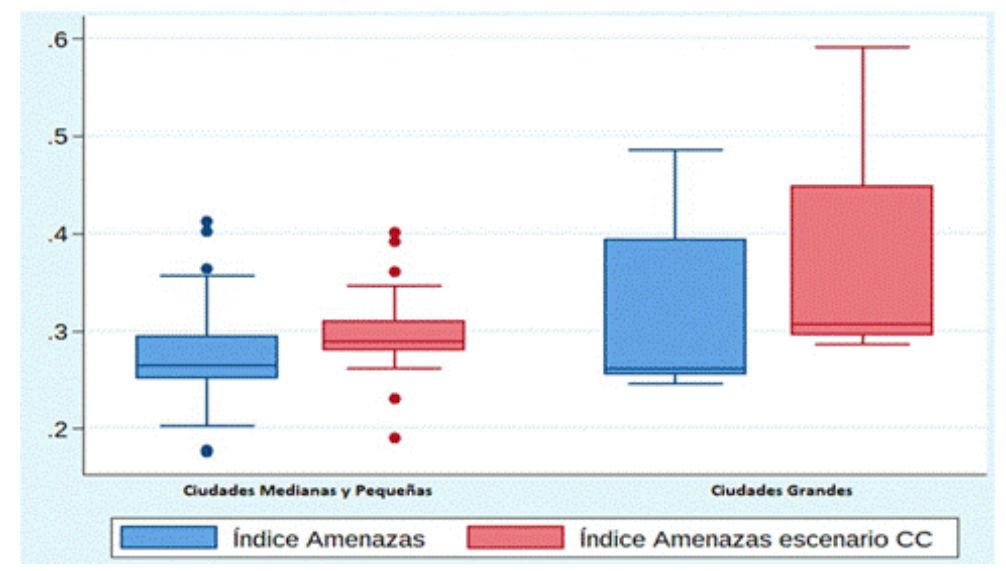

GRÁFICO 2

Distribución del Índice de Amenazas Climáticas por tamaño de los municipios Fuente: elaboración propia con base en escenarios estimados con datos de DESINVENTAR (2015).

En función de la metodología propuesta, la información climática y el análisis de eventos extremos declarados por Bolivia con base en DESINVENTAR (2015), se generó un índice de amenazas de los eventos climáticos, en las áreas urbanas de Bolivia, en este caso inundaciones, deslizamientos de tierra, sequías y granizadas; tanto para los eventos actuales como para la generación de escenarios climáticos futuros ${ }^{[3]}$.

Entre los principales resultados del índice de amenazas se destaca que: por un lado, los municipios más afectados por el aumento de las sequías serán los pertenecientes a los departamentos de Chuquisaca, Potosí y Tarija, dado que poseen actualmente zonas secas y cálidas, y el aumento de la temperatura ocasionará poca 
accesibilidad a fuentes de riego y agua para consumo humano. Por otro lado, los municipios del altiplano se verán afectados por el deshielo de los glaciares, ver mapa 1.

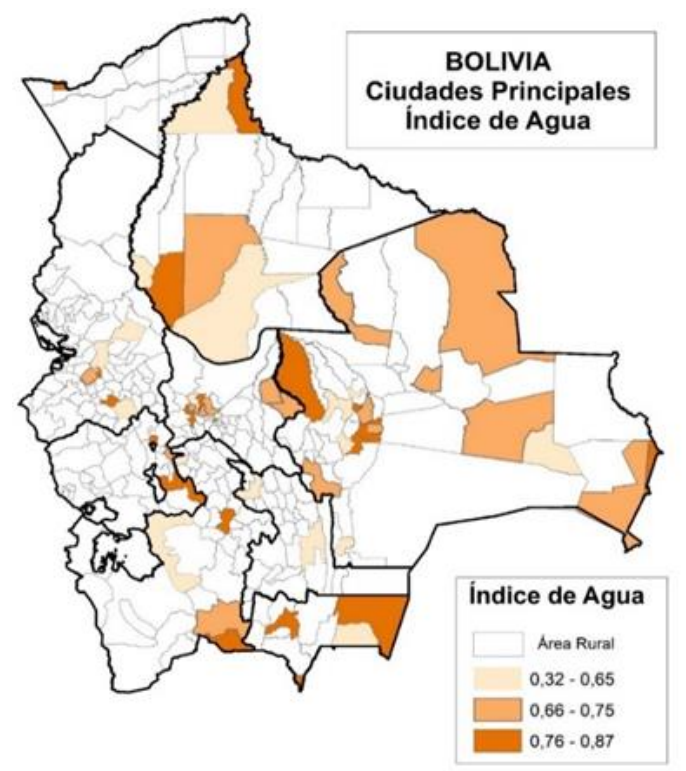

MAPA 1.

Dimensión de peligros y Amenazas

Fuente: elaboración propia

En particular, el índice de amenazas muestra que la sequía y escasez de agua se han convertido en un fenómeno recurrente en Bolivia en la última década y la situación es especialmente pronunciada desde 2015. También sufre los efectos del cambio climático en forma de períodos secos más largos y recurrentes, lluvias cortas e intensas, y mayor frecuencia de granizadas y heladas. Esta situación se ve exacerbada por la degradación de los recursos naturales. En el otro extremo, se observa que dada la riqueza geográfica de la zona oriental que posee territorio amazónico y chiquitano las sequías no golpearan muy fuerte a esta zona por lo que presentan un índice de amenazas más bajo, sin embargo, se debe recordar que a pesar de las características húmedas de estas zonas en el 2019 se vivió el incendio más devastador de la historia moderna.

\subsection{Indice de vulnerabilidad al cambio climático (IVCC)}

Después de generar el IVCC se realizó una categorización con 3 grupos $\left.^{[4]}: 1\right)$ municipios con baja vulnerabilidad, son los que tienen un IVCC menor a 0.33 ; 2) los municipios que van de 0.33 a 0.48 se consideran de vulnerabilidad media; y 3 ) los municipios que tienen un IVCC mayor a 0.48 son municipios con alta vulnerabilidad.

Tal como se observa en el mapa 2, la gran parte de la vulnerabilidad está ubicada en los municipios con menor número de habitantes, esto se debe, en principio, a los altos niveles de pobreza, la baja capacidad institucional y la mayor presencia de grupos vulnerables, y en algunos casos la ubicación en zonas de alto riesgo, ver características de los municipios por región de análisis en anexos (IMCO 2012). Otra característica que llama la atención es que los municipios de la región de los llanos tropicales (Beni, Santa Cruz y parte de Cochabamba) que presentan mayor vulnerabilidad al margen de ser municipios con poca densidad demográfica son municipios que presentan problemas de almacenamiento de agua, dado que la mayor cantidad de sus reservas son superficiales. Mientras que los municipios ubicados en la región altiplánica que presentan mayor vulnerabilidad son los municipios de Potosí, y aquellos con mayores niveles de pobreza. 


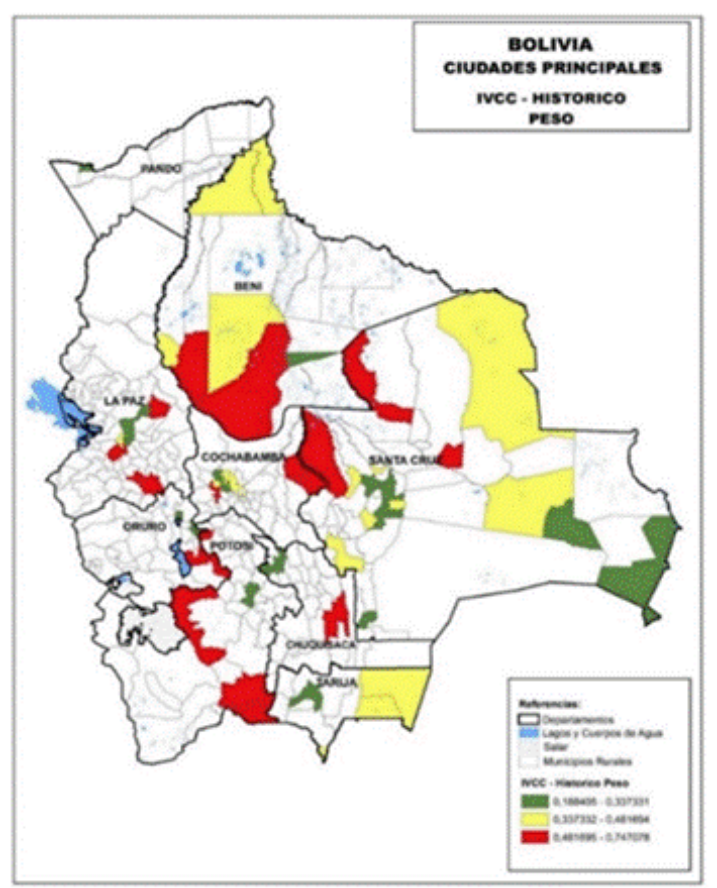

MAPA 2.

ndice de Vulnerabilidad Climática datos históricos con pesos estadísticos Fuente: elaboración propia con base en IVCC

Con relación a los municipios más poblados, que en este caso comprenden las capitales de departamento, a excepción de Cobija, se observa que estas unidades tienen índices relativamente mejores que los municipios con ciudades pequeñas e intermedias; sin embargo, se debe tener en cuenta que poseen mayor probabilidad de daños en situaciones de desastres ambientales, dada la mayor cantidad de habitantes y los bajos niveles de resiliencia de estas ciudades. Los municipios más poblados suelen a su vez tener mejores planes de ordenamiento territorial por lo que la ubicación de sus zonas más vulnerables, están identificadas en una primera instancia.

Un ejemplo de lo anterior es la tormenta de granizo del 19 de febrero de 2002 en la ciudad de La Paz, que provocó muchas inundaciones y deslizamientos de tierra, ocasionando a su vez importantes estragos, los recuentos enumeran al menos 69 muertos, 130 heridos y 50 desaparecidos. Los daños estructurales fueron evaluados en 10 millones de dólares (daños en las vías de comunicación, los vehículos, los edificios públicos y privados como el centro de salud); además de las interrupciones de alimentación en energía eléctrica y en agua potable que agravaron los funcionamientos de la fase de urgencia (Villegas, 2002;GMLP, 2007).

A esto se suma que, aproximadamente 200 familias se vieron obligadas a abandonar sus viviendas damnificadas (Combaz, 2007; Hardy, 2009). Después de este incidente la alcaldía de la ciudad de La Paz diseñó un plan de riesgos y reforzó su unidad de emergencias, siendo el primer municipio en contar con una normativa de esas características, posteriormente en el año 2014 el gobierno promulgó la ley $\mathrm{N}^{\circ} 602$ "Ley de Gestión de Riesgos", a partir de dicha normativa las municipalidades están obligadas a poseer un plan de riesgo ante contingencias ambientales y emergencias, sin embargo, solo 7 municipios de 9 municipios capitales cuentan con sus respectivos planes. Los planes, a su vez, dependen de la capacidad municipal para poder implementar las disposiciones de la ley y generar los mecanismos adecuados de planificación y reacción ante contingencias, lo cual no ha figurado como un eje de política pública para la gestión de riesgos. Ante ello, la vulnerabilidad radica en la incapacidad de identificación, movilización, mitigación y recomposición de los daños hechos por las contingencias citadas. Por ende, la construcción del índice de amenazas puede contribuir 
a identificar las zonas en donde estos riesgos han presentado históricamente mayor rezago e impactos por los fenómenos de variaciones de precipitación y temperatura.

Todas las características antes mencionadas se integran en la dimensión de capacidad sectorial (NoveloCasanova y Suarez, 2015) y sensibilidad institucional que, tal como se observa en los cuadros 1 y 2 , juntas aportan más del 83\% de la variabilidad del IVCC, por lo tanto, la participación del estamento público en todas sus aristas es de vital importancia al momento de mitigar los cambios climáticos; además, la capacidad de gestión de los diferentes sectores (agua, energía, riesgo, agrícola, ambiental y transporte ${ }^{[5]}$ ) se deben convertir en los ejes de las políticas públicas futuras.

\section{CUADRO 1}

Dinámica de ponderadores estadísticos del IVCC

\begin{tabular}{lc}
\hline Peligros y Amenazas & $17.64 \%$ \\
Capacidad institucional & $76.13 \%$ \\
Capacidad sectorial & $6.23 \%$ \\
\hline
\end{tabular}

Fuente: elaboración propia, usando la descomposición de Shapley

CUADRO 2

Ponderadores estadísticos del subíndice de capacidad sectorial del IVCC

\begin{tabular}{lc}
\hline Subindice agna & $40.00 \%$ \\
Subindice enetgía & $21.00 \%$ \\
Subindice tiesgo & $14.40 \%$ \\
Subindice agnicola & $13.90 \%$ \\
Subindice ambiente & $7.60 \%$ \\
Subindice transponte & $3.10 \%$ \\
\hline
\end{tabular}

Fuente: elaboración propia, utilizando la descomposición de Shapley, (1953)

Al realizar la descomposición de Shapley a los subíndices de la dimensión de capacidad sectorial (cuadro 3) observamos que el componente que aporta más variabilidad a la dimensión es el subíndice de agua con 40\%. Es decir que el 40\% de la dimensión de capacidad sectorial sectores de alta relevancia estratégica son el de salud (Cortinovis 1993) y el resto es explicada por el componente agua.

Por lo tanto, en los siguientes acápites se hace un análisis de esta categoría con el fin de identificar los efectos del cambio climático en el sector agua y sus posibles impactos sociales (Cutter et al, 2009; Cutter et al, 2003).

\subsection{Capacidad sectorial del agua en Bolivia}

En Bolivia la propagación de la urbanización, la degradación de las cuencas fluviales, y la contaminación por actividades de desarrollo amenazan la seguridad del agua en las zonas urbanas. Lo anterior sumado a los altos niveles de vulnerabilidad debido al desarrollo insuficiente de la infraestructura en los asentamientos informales y la alta fragmentación del suministro básico de agua y la prestación de servicios de saneamiento con una mirada de operadores locales de agua, aumentan aún más los desafíos del agua en las ciudades de Bolivia (World Bank, 2019), ver mapa 3. 


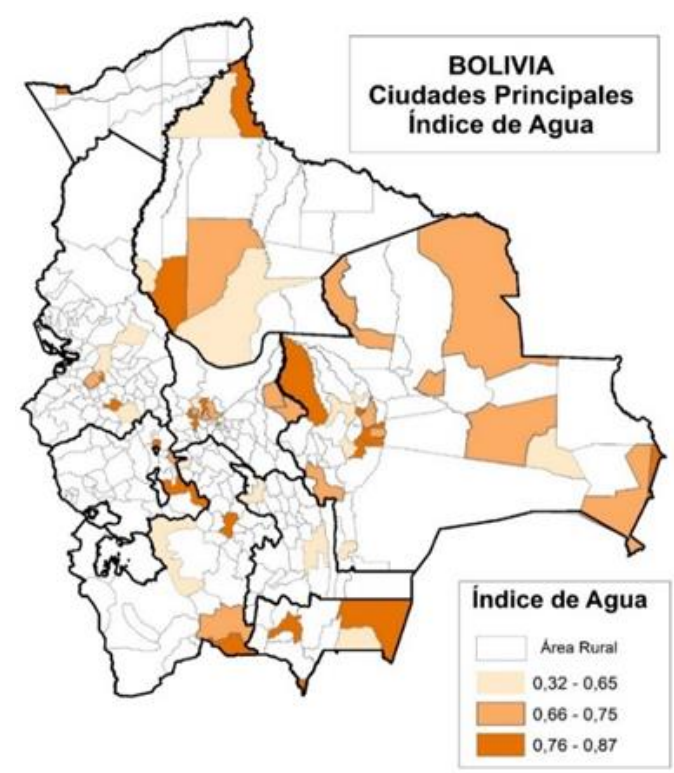

MAPA 3.

Índice de capacidades del sector Agua

Fuente: elaboración propia

$\mathrm{Al}$ margen de contar con recursos hídricos, la desigual distribución de estos ocasiona que se genere una demanda no satisfecha de este recurso en algunas zonas de Bolivia. El estrés hídrico es particularmente desafiante en partes específicas del país, especialmente en ciudades más pequeñas. Cabe aclarar que, al margen que las grandes ciudades de Bolivia cuentan con una cobertura, mayor que los municipios más pequeños, esta no llega al 100\% de sus habitantes, tal como menciona en Sanders, K; Miranda, J y Olivera, S. (2019) y en el estudio del Banco Mundial (2019) las ciudades metropolitanas del eje troncal poseen una cobertura diferencial siendo el área metropolitana de Santa Cruz, dado que el $98 \%$ de su población cuenta con servicios de agua y el $94 \%$ de esta recibe agua desinfectada a través de cloración. El área metropolitana de La Paz posee una cobertura del 92.3\%, mientras que Cochabamba presenta una cobertura del $89 \%$, y el 27\% de sus usuarios no cuentan con el servicio las 24 horas del día, estos datos muestran el grado de vulnerabilidad de esta ciudad.

En este orden de ideas es importante recapitular la importancia de conocer la vulnerabilidad en ciertas zonas a partir de la interrelación del manejo de los recursos hídricos, la provisión de servicios y la resiliencia del sector agua ante el cambio climático, con el fin de coadyuvar a la formulación de políticas sectoriales integrales, sobre todo aquellas que pueden priorizar zonas urbanas para el manejo de contingencias de inundaciones y sequías.

Para realizar el índice de vulnerabilidad del sector se analizaron las variables de: cobertura de agua potable, alcantarillado, fuente de agua potable -superficial y subterránea-, así como gastos en inversión y mantenimiento del sector a nivel municipal (MEF/MPD 2017). Con tres categorías ${ }^{[6]}$ donde los municipios con menos de 0.65 puntos del índice están en mejor capacidad del sector agua, los municipios de 0.66 a 0.75 poseen capacidad media y los que tienen un valor superior a 0.76 son municipios con baja capacidad sectorial.

Tal como se observa en el mapa 3 la región de los llanos (Beni, Santa Cruz y Cobija), del altiplano (La Paz, Oruro y Potosí) y el chaco (Tarija) presentan niveles de capacidad institucional ${ }^{[7]}$ más bajos, esto se refleja en su baja capacidad de almacenamiento de agua potable. Los municipios con mayor vulnerabilidad en el sector agua poseen los siguientes elementos en común, baja cobertura de sistemas de alcantarillado y prácticamente ningún sistema de tratamiento de aguas residuales, abastecimiento de agua potable de una sola fuente de agua- ya sea superficial o subterránea- y, bajos niveles de mantenimiento e inversión en el sector. Además, el sector muestra una heterogeneidad consistente en el desarrollo y la capacidad institucional de cada municipio 
dado que está altamente condicionado a la generación de recursos propios de las alcaldías y la capacidad de planificación y ejecución de medidas de emergencia de los municipios.

Tal como se observa en el cuadro 3 al realizar la descomposición de Shapley, (1953) se observa que el indicador que aporta mayor variabilidad es la fuente de agua potable de los municipios, ya que si es una fuente superficial es muy vulnerable a sequías, deslizamientos e inundaciones. Por otra parte, en relación con el uso de agua subterránea, la falta de estudios sobre la calidad de estas aguas y la existencia de reservas en los acuíferos hacen del indicador un punto que requiere un análisis a más profundidad. A su vez la extracción de aguas de los acuíferos requiere una integración a los sistemas de generación eléctrica o de transporte de combustibles que incrementan la vulnerabilidad del sector en el caso de cortes en el suministro eléctrico o derrumbes en el sistema de carreteras. La falta de integración y coordinación del sector agua con otros sectores de desarrollo como el eléctrico o el de transporte, aumenta la proporción y magnitud de los impactos negativos de las contingencias y la vulnerabilidad.

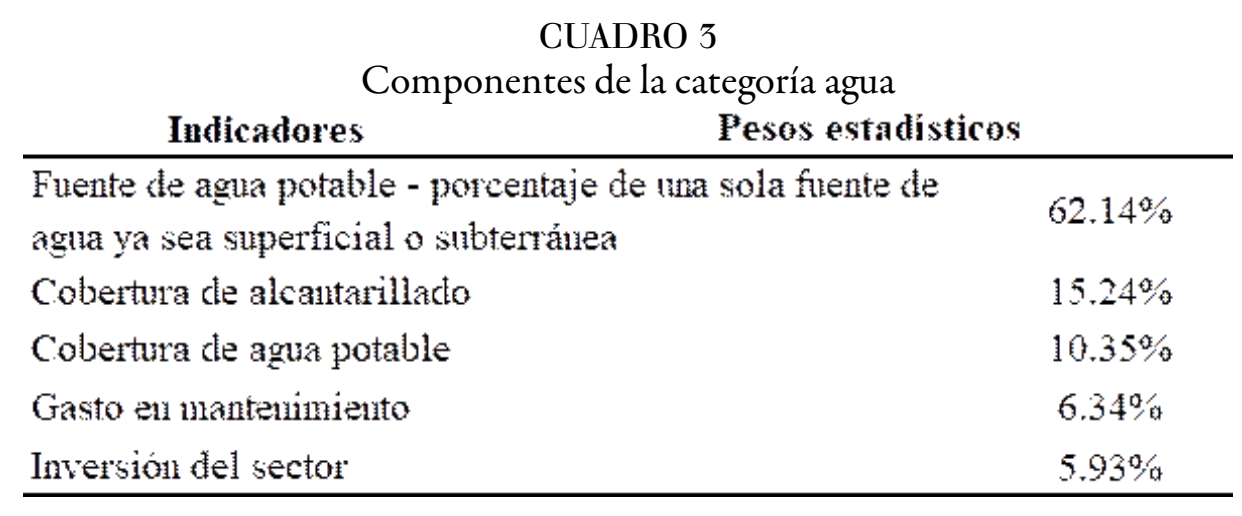

Fuente: elaboración propia

Los datos muestran que la dependencia a una sola fuente de agua ya sea superficial o subterránea, varía según el tamaño de los municipios. En este sentido, las áreas urbanas de Cochabamba y Santa Cruz presentan estrategias de acceso a fuentes de agua más diversificadas; mientras que municipios capitales como: La Paz, Sucre y Potosí usan mayormente fuentes de agua superficial, incrementando su vulnerabilidad ante eventos extremos como sequías prolongadas. En similar situación se encuentra la ciudad de El Alto, dado que depende en un $100 \%$ de sus fuentes superficiales provenientes de los nevados de este municipio, debido al calentamiento global y el deshielo acelerado de los nevados se encuentra en un escenario de mayor vulnerabilidad, a esto se debe sumar que es la ciudad más joven y con la mayor tasa de crecimiento poblacional, en este orden de ideas es importante que las autoridades locales y nacionales generen mecanismos de resiliencia en esta ciudad (ver mapa 4 ). 


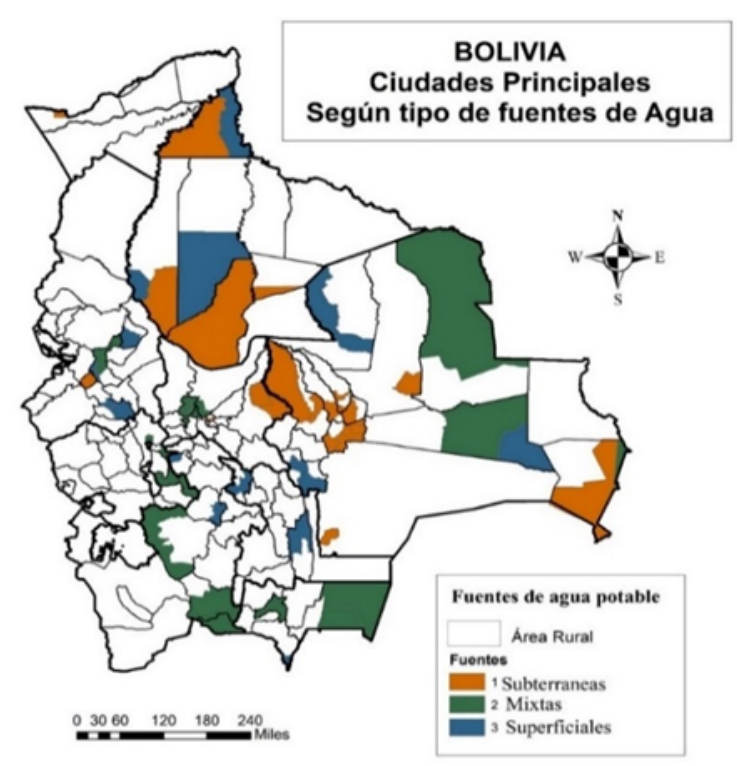

MAPA 4

Distribución de las fuentes de agua

Fuente: elaboración propia

Con relación a los departamentos que, por sus condiciones topográficas, dependen casi exclusivamente de fuentes subterráneas se destacan los municipios de Santa Cruz y Oruro, ahí la calidad del agua y la disponibilidad real de los acuíferos es una limitante que debe ser considerada en el manejo de este recurso. Finalmente, si analizamos otros municipios urbanos como los de la cuenca del río Amazonas, observamos que por razones topográficas y de calidad del agua la mayor parte de los municipios opta por las fuentes de agua subterránea a excepción de tres municipios que cuentan con sistemas de colinas o diferenciales de pendientes que les permiten usar aguas superficiales. Los datos mencionados anteriormente son a nivel municipal, con el fin de realizar un análisis más detallado de las fuentes de agua se decidió describir la situación del acceso al agua potable de los hogares bolivianos, con base en la Encuesta de Hogares 2018, detallado en el Cuadro 4. 
CUADRO 4

Fuente de agua potable en los hogares, Bolivia $2018^{[8]}$

\begin{tabular}{lll}
\hline Tipo & Frecuencia & Porcentaje \\
\hline $\begin{array}{l}\text { Cañería de red dentro } \\
\text { de la vivienda }\end{array}$ & $1,245,785.30$ & 35.95 \\
$\begin{array}{l}\text { Cañería de red fuera de } \\
\text { la vivienda, }\end{array}$ & $1,042,385$ & 30.08 \\
$\begin{array}{l}\text { Pileta pública } \\
\text { Cosecha de agua de }\end{array}$ & $18,982.73$ & 0.55 \\
lluvia & $10,054.62$ & 0.29 \\
$\begin{array}{l}\text { Pozo perforado o } \\
\text { entubado, con bomba }\end{array}$ & $117,996.48$ & 3.41 \\
$\begin{array}{l}\text { Pozo excavado } \\
\text { Cubierto, con bomba }\end{array}$ & $98,760.09$ & 2.85 \\
$\begin{array}{l}\text { Pozo excavado } \\
\text { Cubierto, sin bomba }\end{array}$ & $72,499.13$ & 2.09 \\
$\begin{array}{l}\text { Pozo excavado no } \\
\text { cubierto }\end{array}$ & $71,608.22$ & 2.07 \\
$\begin{array}{l}\text { Manantial o Vertiente } \\
\text { protegida }\end{array}$ & $352,274.27$ & 10.17 \\
$\begin{array}{l}\text { Riego/Acequia/Vertiente } \\
\text { no protegida }\end{array}$ & $280,362.71$ & 8.09 \\
$\begin{array}{l}\text { Agua embotellada } \\
\text { Carro repartidor }\end{array}$ & $54,199.25$ & 1.56 \\
$\begin{array}{l}\text { (Aguatero) } \\
\text { Otro (Especifique) }\end{array}$ & $88,860.81$ & 2.56 \\
\hline Total & $11,095.05$ & 0.32 \\
\hline
\end{tabular}

Fuente: elaboración propia con base en EH 2018

A pesar de las políticas públicas y programas que se generaron, como ser Mi Agua, Agua y Riego PROAR $\left.{ }^{[9}\right]$, entre otros que se desarrollaron a nivel departamental y municipal a favor de la cobertura total de agua potable y alcantarillado, se observa que solo el $35.9 \%$ de los hogares poseen una cañería de red dentro de sus viviendas, mientras que cerca del $29 \%$ acceden al agua mediante fuentes naturales, como: pozos, manantiales, cosecha de agua de lluvia, riego, acequia y vertientes; es decir, en momentos de sequía estos hogares son los más vulnerables respecto a la obtención de este elemento, razón por la cual se deben generar políticas más rigurosas con el fin de garantizar el derecho al agua potable y alcantarillado a todos los bolivianos.

\subsection{Propuesta de politicas públicas}

Como se observó, es importante desarrollar diferentes políticas públicas que ayuden a mitigar los efectos del cambio climático y reforzar la capacidad del sector agua en el país, por lo tanto, se realiza una recopilación de los principales programas implementados por el gobierno; además, con base en las proyecciones ambientales desarrolladas en el acápite anterior y el índice de capacidad del sector agua, se realiza un resumen de las principales propuestas de políticas públicas desagregadas según división regional ${ }^{[10]}$.

En primer lugar, se debe reconocer que en los últimos años el gobierno invirtió en promedio cerca del 0.55\% del PIB nacional en el sector del agua, es decir alrededor de 18.924 millones de bolivianos, entre 2006 y 2016. Estos recursos se distribuyeron en: agua potable y saneamiento básico ( 9.785 millones), recursos hídricos (2.154 millones), riego (5.129 millones) y drenaje pluvial (1.856 millones) (MEFP, MPD, 2017). Los principales programas y proyectos que se generaron fueron:

1) Programa Mi Agua, implementado desde el año 2007, este programa cuenta con 5 versiones y tiene el objetivo de financiar proyectos de agua potable en pequeñas comunidades del medio rural. 
2) Programa Mi Riego, implementado desde el año 2008 este programa cuenta con 4 versiones y tiene el objetivo de financiar proyectos de riego en pequeñas comunidades del medio rural

3) Programas de agua potable y saneamiento para pequeñas localidades y comunidades rurales de Bolivia (APCR) y el programa de agua potable y saneamiento destinado a las áreas urbanas más pequeñas y pobres del país (APPC).

4) Proyecto de agua y alcantarillado periurbano en sus tres fases (PAAP) y (URBANO) este último con financiamiento del Banco Mundial.

5) Programa de agua, saneamiento y drenaje en áreas rurales (PASAP) y (PASAR).

6) Programa de Agua y Riego (PROAR), destinado al financiamiento de proyectos de agua potable, saneamiento y riego de mediano tamaño y a nivel nacional.

7) Programa de Agua, Saneamiento, Residuos Sólidos y Drenaje Pluvial (PROASRED), el cual ha sido diseñado para financiar una canasta de proyectos de tamaño mediano y pequeño de agua potable, alcantarillado, depuración de aguas servidas y gestión de residuos sólidos.

8) Programas de Saneamiento de lagos y ríos como: programa del Lago, destinado al saneamiento del Lago Titicaca; programas PSLT y 3PTARS proyectos de remediación ambiental del río Rocha;

9) Programas de cosecha de agua

10) Programa nacional de agua subterránea

11) Programas de tratamiento de aguas subterráneas PTARS

12) Programa de uso eficiente del agua y adaptación al cambio climático

En paralelo a los programas implementados, el gobierno promulgó diferentes leyes y decretos a favor del uso del agua, entre los que se destaca: la Ley No. 300, Ley Marco de la Madre Tierra y Desarrollo Integral para Vivir Bien, el Plan Nacional de Cuencas, el Programa Plurianual de Gestión Integrada de Recursos Hídricos y Manejo Integral de Cuencas 2013-2017; el Plan Nacional de Riego para Vivir Bien; la Agenda de Riego 2025 (establece las metas e indicadores a cumplir en el marco de la Agenda Patriótica); el Plan Sectorial de Desarrollo de Saneamiento Básico 2016 -2020, entre otros.

Sin embargo, a pesar de los avances institucionales y los esfuerzos fiscales del gobierno la capacidad de almacenamiento del país es una de las más bajas de la región; tal como menciona la CAF (2017) "Bolivia solo cuenta con una capacidad de almacenamiento en embalses de unos 56 por habitante, cuando Ecuador y Perú, con geografías y climas equivalentes, disponen de $489 \mathrm{~m}^{3}$ y 190 , respectivamente" (p.22).

A lo anterior se suman las demandas específicas de las diferentes regiones del país. Entre las que se destacan: En la zona del altiplano por sus características geográficas, la fuente de agua más utilizada (con excepción de la ciudad de Oruro) es la superficial proveniente de los nevados, sin embargo, dado el aumento de la temperatura se proyecta que en menos de 50 años los nevados desaparezcan, por lo que es necesario priorizar políticas públicas de almacenamiento de agua, diversificación de fuentes de agua y ampliación de las redes de distribución de agua potable a todos los hogares. Por otro lado, se deben generar medidas defensivas y muros de contención para controlar las inundaciones y deslizamientos de tierra, en el 2002 la ciudad de La Paz vivió una de las más grandes tragedias por inundación que se podía haber evitado si este municipio hubiera contado con: 1) plan de riesgo; 2) retén de emergencias; 3) sistema de alcantarillado más amplio y limpio; y, 4) una cultura de riesgo, donde la población sepa qué hacer en caso de inundaciones, ver tabla 4. 
TABLA 4.

Fuente: elaboración propia

\begin{tabular}{|c|c|c|c|c|c|}
\hline \multirow{4}{*}{ 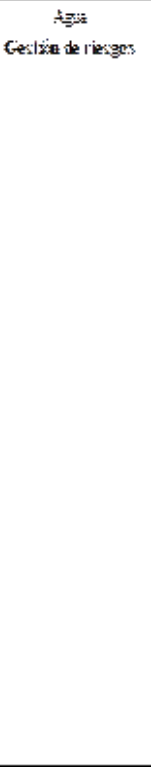 } & \multirow{2}{*}{ 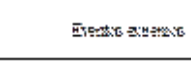 } & \multirow{2}{*}{ 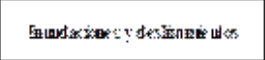 } & 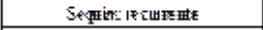 & 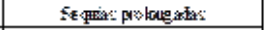 & \multirow{2}{*}{ 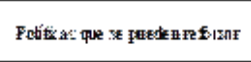 } \\
\hline & & & 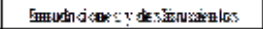 & 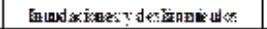 & \\
\hline & Eoticas rativizistas & 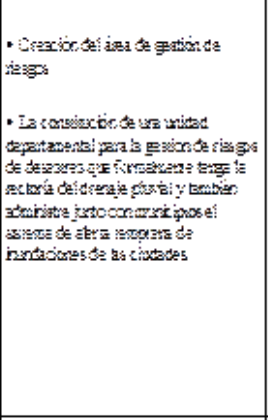 & 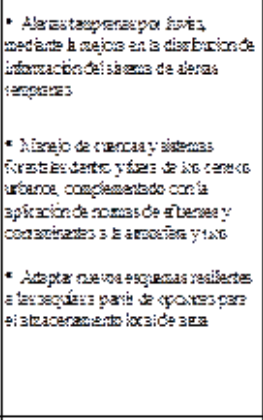 & 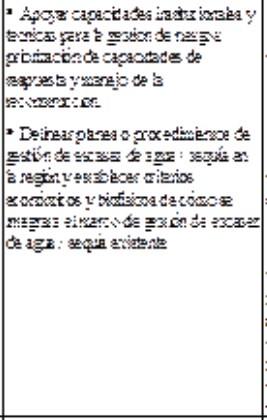 & 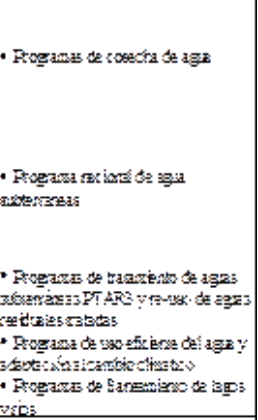 \\
\hline & Naxpoda trastax tra & 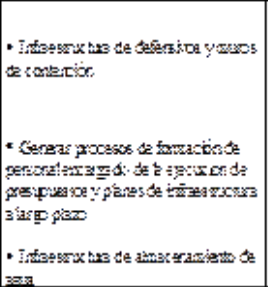 & 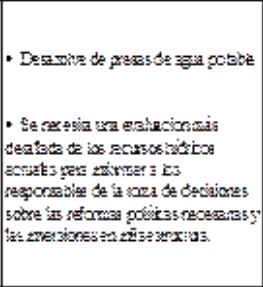 & 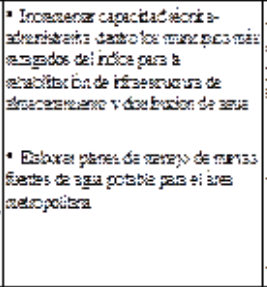 & 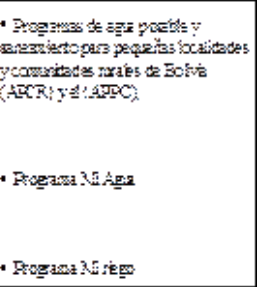 \\
\hline
\end{tabular}

En relación con los municipios con ciudades medianas, intermedias y pequeñas del Altiplano se destacan políticas de gestión de riesgo y distribución de agua potable, además se deben centrar en la aplicación de políticas para el manejo del alcantarillado y saneamiento, priorizando su planificación urbana para la ampliación de sus redes de distribución.

En la región de los valles, en los municipios con ciudades intermedias y pequeñas la gestión del riesgo por inundaciones y deslizamientos y el manejo de agua potable son prioritarios, dado que la región en su conjunto va de tendencias climáticas cálidas y húmedas a una tendencia futura de cálido a seco, con extremos de lluvias en los meses de verano y en condiciones de fenómenos como el Niño o la Niña, por lo que se debe priorizar la creación de presas de agua potable, sistemas de baterías de pozos de agua potable, entre otros.

La zona metropolitana de Cochabamba y los municipios de Sucre y Tarija presentan problemáticas semejantes, ver tabla 5, sin embargo, a nivel específico se destaca:

1) En la ciudad de Sucre, dado las fuertes granizadas que esta ciudad presenta, en el futuro se debe priorizar la creación de infraestructura adecuada para este tipo de eventos. Además, al ser un municipio que depende en totalidad de fuentes de agua superficiales y tener mayor nivel de amenaza por sequías, es primordial generar sistemas de almacenamiento y diversificación de sus fuentes de agua.

2) En relación con la zona metropolitana de Cochabamba se debe mejorar el sistema de alcantarillado y diversificar las fuentes de almacenamiento de agua potable.

3) Finalmente, en el caso de Tarija se debe mejorar el tratamiento de aguas residuales y generar un saneamiento del río Rocha. 
TABLA 5.

Priorización de políticas públicas región de los Valles

(departamentos de Cochabamba, Chuquisaca y Tarija)

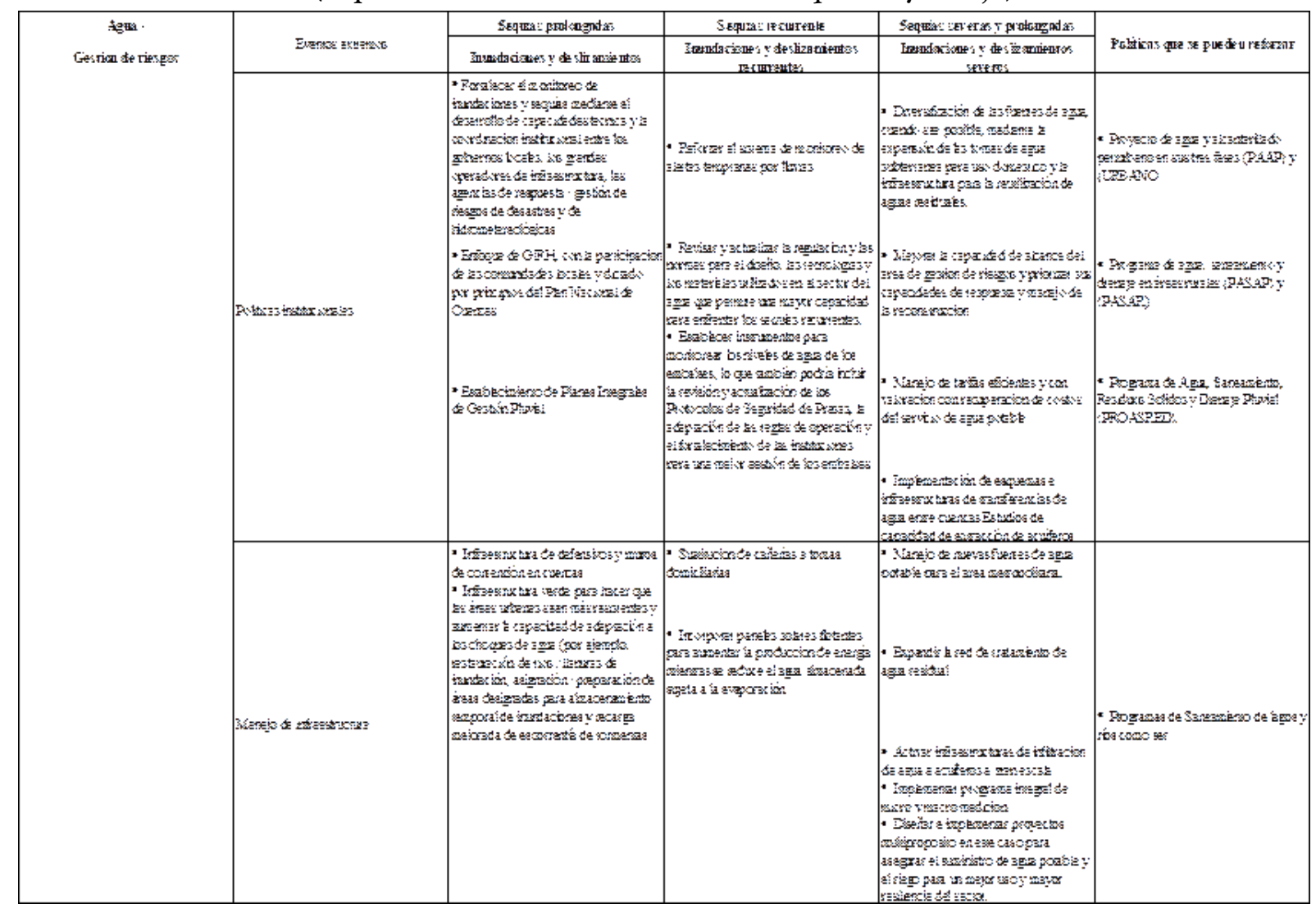

Fuente: elaboración propia

En la región tropical del país la ciudad de Santa Cruz tiene, al igual que el resto de la región, un cambio de clima cálido - húmedo a cálido - seco para mediados de siglo, lo que incrementa la tendencia de sequías, incendios y de eventos extremos en los meses de verano. Por lo que la gestión de riesgo y manejo de agua son los sectores prioritarios para esta región. En relación con los municipios de los departamentos del Beni y Pando, al margen de pertenecer a la cuenca del Amazonas y en promedio contar con mayor disponibilidad de agua se deben generar políticas de infiltración de a acuíferos, almacenamiento, tratamiento de las aguas superficiales e infraestructura de defensivos y muros de contención de cuencas, ver tabla 6 . 
TABLA 6.

Priorización de políticas públicas región oriental (departamentos de Santa Cruz, Beni y Pando)

\begin{tabular}{|c|c|c|c|c|c|}
\hline \multirow{4}{*}{$\begin{array}{l}\text { Agua - } \\
\text { Gestizan to résgors }\end{array}$} & \multirow{2}{*}{ 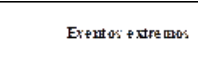 } & 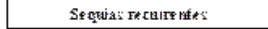 & 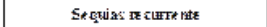 & 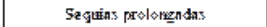 & \multirow{2}{*}{ 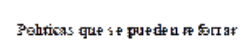 } \\
\hline & & Inmadariones & Immokacisuts & Immada kiones: & \\
\hline & 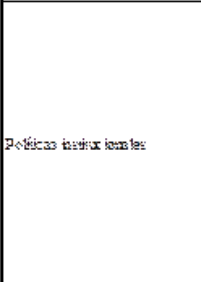 & 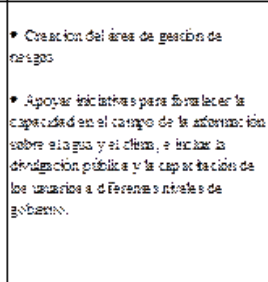 & 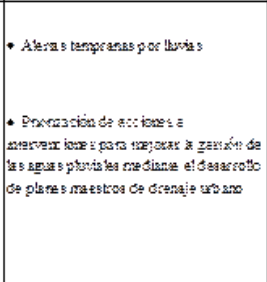 & 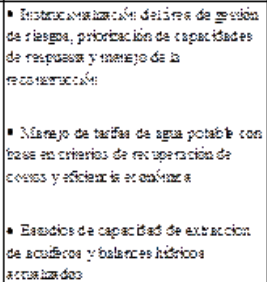 & 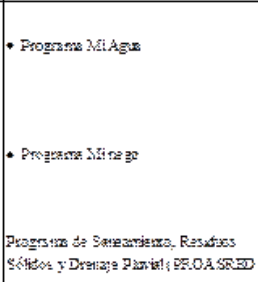 \\
\hline & 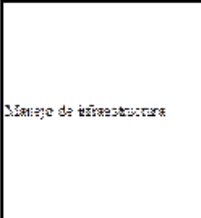 & 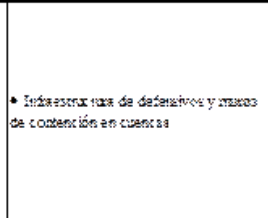 & 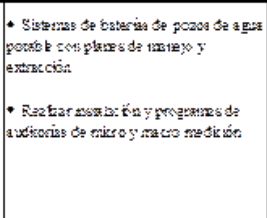 & 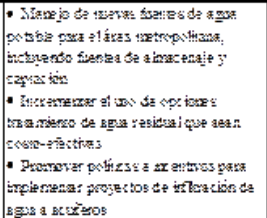 & 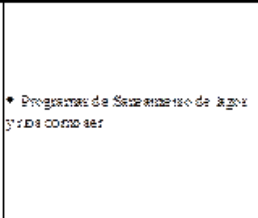 \\
\hline
\end{tabular}

Fuente: elaboración propia

A su vez, si analizamos la región de los municipios urbanos de la cuenca baja del río Amazonas, nos encontramos con deficiencias en la gestión de riesgos (Brooks, 2003) en especial en la reconstrucción de infraestructura dañada, una serie de carencias en estudios de fuentes de agua potable y su tratamiento, así como un bajo desempeño en la gestión de redes de electrificación en estos municipios, temas que deben ser tratados para evitar futuras complicaciones.

A lo anterior se suma el incremento en las sequías a nivel nacional lo cual genera un panorama nada favorable para los municipios que poseen altos niveles de vulnerabilidad respecto a este elemento. Además, cuando se examinan resultados sectoriales, como son los incrementos de conexiones, la calidad sanitaria del agua que llega a los hogares, la continuidad del servicio, el cuidado de la gestión del recurso hídrico y la consideración a las consecuencias del cambio climático aún queda un vasto camino por recorrer. Finalmente, se debe recordar que el acceso al agua es un derecho humano por lo que debe ser un tema prioritario para el desarrollo social y económico de los municipios y del país en general.

\section{Conclusiones}

Para generar políticas públicas específicas se debe crear un índice de vulnerabilidad de todos los municipios de la región, para ello se necesita mejores fuentes de información que capten las necesidades a nivel municipal.

Además, según el método de descomposición que se aplicó al IVCC se observa que el componente de capacidad sectorial, conformada por: el nivel económico de los municipios, grupos vulnerables y capacidad institucionales, es el que más aporta en la explicación de la vulnerabilidad de los municipios. En este sentido, es importante asegurar una inversión pública eficaz y eficiente para aumentar la capacidad de resiliencia de la infraestructura desarrollada.

Por otro lado, los efectos del cambio climático se visualizarán en el aumento de las sequías y deslizamiento de tierra, por lo que es necesario generar políticas de almacenamiento y distribución eficiente del agua.

Donde el sector agua es el que aporta más a la variabilidad del índice de vulnerabilidad al cambio climático, por lo que destaca la importancia de generar políticas públicas de mejora de este sector, además se debe reconocer que el derecho al acceso a fuentes de agua potable es un derecho de la humanidad reconocido en diferentes tratados internacionales.

Finalmente, cabe recordar que Bolivia tiene un lugar privilegiado en la obtención de agua dulce, dado que por su territorio pasan dos de las tres cuencas de Sudamérica, sin embargo, posee el nivel de almacenamiento más bajo de la región, lo que aumenta sus niveles de vulnerabilidad que se incrementa por el aumento de las 
sequías. Además, al aceptar que el acceso al agua es uno de los principales derechos humanos, el gobierno central tendrá que generar políticas públicas de gestión de este recurso, como: aumento de la cobertura de riego, saneamiento básico, medidas de almacenamiento de agua, diversificación de las fuentes de agua potable y sobre todo asegurar el acceso de todos los habitantes del país al agua potable con el fin de contribuir al desarrollo social y económico de estos municipios y con ello asegurar el desarrollo del país en general.

\section{LITERATURA CITADA}

Adger, W., Brooks, N., Bentham, G., Agnew, M., \& Eriksen, S. (2004). New indicators of vulnerability and adaptative capacity. Tyndall Centre. https://www.ipcc.ch/apps/njlite/ar5wg2/njlite_download2.php?id=7785

Bai, X, R Dawson, D Ürge-Vorsatz, G Delgado, A Barau, S Dhakal, D Dodman, L Leonardsen, V Masson-Delmotte and D Roberts (2018), "Six research priorities for cities and climate change", Nature Vol 555, No 7694, pages 23-25. https://doi.org/10.1038/d41586-018-02409-z

Barnett, J., Lambert, S., \& Fry, I. (2008). The hazards of indicators: insights from the environmental vulnerability index. Annals of the Association of American Geographers, 98(1), 102-119. https://www.tandfonline.com/doi/ abs/10.1080/00045600701734315 https://doi.org/10.1080/00045600701734315

Beccari B., (2016) A comparative analysis of disaster risk, vulnerability and resilience composite indicators, PLoS Curr. Disasters 8 1-53 Edition 1.https://pubmed.ncbi.nlm.nih.gov/27066298/ https://doi.org/10.1371/ currents.dis.453df025e34b682e9737f95070f9b970

Becker, M. Saisana, P. Paruolo \& I. Vandecasteele, (2017) Weights and importance in composite indicators: closing the gap, Ecol. Indicat. 80. https://www.sciencedirect.com/science/article/pii/S1470160X17301759 https:// doi.org/10.1016/j.ecolind.2017.03.056

Brooks, N. (2003). Vulnerability, risk and adaptation: A conceptual framework. Tyndall Centre. https:// www.ipcc.ch/apps/njlite/srex/njlite_download.php?id=5463

Cortinovis, I., Vella, V., \& Ndiku, J. (1993). Construction of a socio-economic index to facilitate analysis of health data in developing countries. Social science \& medicine, 36(8), 1087-1097. https://www.sciencedirect.com/science/ article/abs/pii/027795369390127P https://doi.org/10.1016/0277-9536(93)90127-P

Cutter, S. L., Boruff, B. J. and Shirley, W. L. (2003), Social Vulnerability to Environmental Hazards*. Social Science Quarterly, 84: 242-261. doi:10.1111/1540-6237.8402002 https://doi.org/10.1111/1540-6237.8402002

Cutter, S., Emrich, C., Webb, J., \& Morath, D. (2009). Social vulnerability to climate variability hazards: A review of the literature. Oxfam America. https://sompiseth.weebly.com/uploads/4/8/4/8/4848831/ literature_review_on_climate_change_vulnerability.pdf

DesInventar: DesInventar (2019)- Inventory system of the effects of disasters, Corporación OSSA, Cali, Colombia, available at: http: //desinventar.org, last access: 10 de enero 2019.

Instituto Mexicano de Competitividad (IMCO). (2012). "Índice de vulnerabilidad Climática". Boletín de prensa. Instituto Mexicano para la Competitividad, A.C. México, D.F. Disponible en: http://imco.org.mx/images/pdf/ Boletindeprensa_IVC_final.pdf

Israeli, O. (2007). A Shapley-based decomposition of the R-square of a linear regression. The Journal of Economic Inequality, 5(2), 199-212. https://ideas.repec.org/a/kap/jecinq/v5y2007i2p199-212.html https:// doi.org/10.1007/s10888-006-9036-6

IPCC: McCarthy, J. J., Canziani, O. F., Leary, N. A., Dokken, D. J., \& White, K. S. (Eds.). (2001). Climate change 2001: impacts, adaptation, and vulnerability: contribution of Working Group II to the third assessment report of the Intergovernmental Panel on Climate Change (Vol. 2). Cambridge University Press. https:// rmets.onlinelibrary.wiley.com/doi/abs/10.1002/joc.775

Mcguire, L., Ford, E., \& Okoro, C. (2007). Natural disasters and older US adults with disabilities: implications for evacuation. Disasters, 49-56. Sagarpa. (15 de noviembre de 2011). Sagarpa. Recuperado el 2011 de noviembre de 2011, de Sagarpa: http://www.sagarpa.gob.mx/saladeprensa/boletines2/paginas/2011B698.aspx https:// doi.org/10.1111/j.1467-7717.2007.00339.x 
McKenzie, D. (2005). Measuring inequality with asset indicators. J Popul Econ 18, 229-260. https://doi.org/10.1007/ s00148-005-0224-7

Mejía Abel; Uzcátegui Germán y Valverde Osvaldo (2017) Agua y Saneamiento en el Estado Plurinacional de Bolivia, Buenos Aires- Argentina, CAF

Ministerio de Economía y Finanzas Públicas \& Ministerio de Planificación del Desarrollo (MEF/MPD) (2017). Inversión del Gobierno Nacional en agua potable, saneamiento básico, recursos hídricos y riego. https://medios.economiayfinanzas.gob.bo/MH/documentos/2016/agosot/ SEPARATA_MIN_ECONOMIA_baja.pdf

Nguefack - Tsague, G., Klasen, S., \& Zucchini, W. (2011). On weighting the components of the human development index: a statistical justification. Journal of human development and capabilities, 12(2), 183-202.https://econpapers.repec.org/article/tafhudca/ v_3a12_3ay_3a2011_3ai_3a2_3ap_3a183-202.htm https://doi.org/10.1080/19452829.2011.571077

Novelo-Casanova D.A. \& G. Suarez, (2015) Estimation of the risk management index (RMI) using statistical analysis, Nat. Hazards 77. https://link.springer.com/article/10.1007/s11069-015-1663-4

OECD, (2008). Handbook on Constructing Composite Indicators: Methodology and User Guide, OECD Publications, Paris.https://www.oecd.org/sdd/42495745.pdf https://doi.org/10.1007/s11069-015-1663-4

Ishizawa, Oscar A., Miranda, J.J., Jiménez, L. F., Villamil, A., Lv, X., Jardillier, R.P.J., \& de Haro López, I., (2017). "Quantitative Analysis of the Impact of Floods in Bolivia," World Bank Other Operational Studies 29043, The World Bank.

Rangecroft,, S., Harrison, S., \& Anderson, K. (2015) Rock Glaciers as Water Stores in the Bolivian Andes: An Assessment of Their Hydrological Importance, Arctic, Antarctic, and Alpine Research, 47:1, 89-98, https:// doi.org/10.1657/AAAR0014-029

Sanders, K; Miranda, J y Olivera, S. (2019) Desarrollando un índice multidimensional de vulnerabilidad a las contingencias climáticas y al cambio climático de los sistemas urbanos. Banco Mundial.

Shapley, L. (1953): A value for n-person games, in: H. W. Kuhn and A. W. Tucker, eds., Contributions to the Theory of Games, Vol. 2 (Princeton University Press). https://doi.org/10.1515/9781400881970-018

Shorrocks, A.F.: Decomposition Procedures for Distributional Analysis: A Unified Framework Based on the Shapley Value (mimeo). University of Essex (1999). http://www.komkon.org/ tacik/science/shapley.pdf

World Bank. (2019). The World Bank Group Action Plan on Climate Change Adaptation and Resilience. Washington, DC: World Bank. http://documents1.worldbank.org/curated/en/519821547481031999/TheWorld-Bank-Groups-Action-Plan-on-Climate-Change-Adaptation-and-Resilience-Managing-Risks-for-aMore-Resilient-Future.pdf

Zaiontz, (2017): Shapley-Owen Decomposition Tools and Applications. https://www.real-statistics.com/multipleregression/shapley-owen-decomposition/

World Bank. 2013 ."Potsdam Institute for Climate Impact Research and Climate Analytics. 2013. Bajemos la temperatura : fenómenos climáticos extremos, impactos regionales y posibidades de adaptación resumen ejecutivo. Washington, DC: World Bank. () World Bank. https://openknowledge.worldbank.org/ handle/10986/14000 License: CC BY-NC-ND 3.0 IGO."

World Resource Institute. 2017. Iceland Water Stress is Helping Drive Conflict and Migration.. https:// www.wri.org/insights/water-stress-helping-drive-conflict-and-migration

DESINVENTAR. 2015. Base de Datos Bolivia. https://www.desinventar.net/DesInventar/profiletab.jsp? countrycode $=$ bol\&continue $=y$

COMBAZ, E., 2007 - Le relogement des populations sinistrées de la municipalité de La Paz. Contextes, évolutions, et impacts des pratiques de relogement de trois catastrophes : la granizada (2002), Llojeta (2003) et German Jordan (1997), 107 pp. Mémoire de Master 1 de Géographie, mention Sciences géographiques, spécialité Mondialisation et développement, Université de Aix-Marseille 1.

GMLP, 2007 - Catálogo de instrumentos en gestión municipal para la reducción de riesgos y preparación ante emergencias, 74 pp.; La Paz: PNUD. 
S. Olivera-Villarroel, et al. La ruleta climática en Bolivia: Vulnerabilidad ante la incertidumbre...

VILLEGAS, S. (ed.), 2002 - Memoria del «Martes Negro». ¡Emergencia por granizada en La Paz!, 59 pp.; La Paz GMLP:, Comisión episcopal de pastoral social CARITAS.

Fundacion de amigos de la Naturaleza. 2019. Memoria (2019) - FAN. https://issuu.com/ fundacionamigosdelanaturaleza/docs/memoria-fan2019_

HARDY, S., 2009 - Explorer la construction de la résilience. Expériences de recherche à La Paz. In: Vulnérabilités sociétales, risques et environnement (A. Pelier \& S. Becerra, eds.): 469-482; París: Éditions L’Harmattan.

\section{Anexo}

\section{Tablas de caracterización de los municipios}

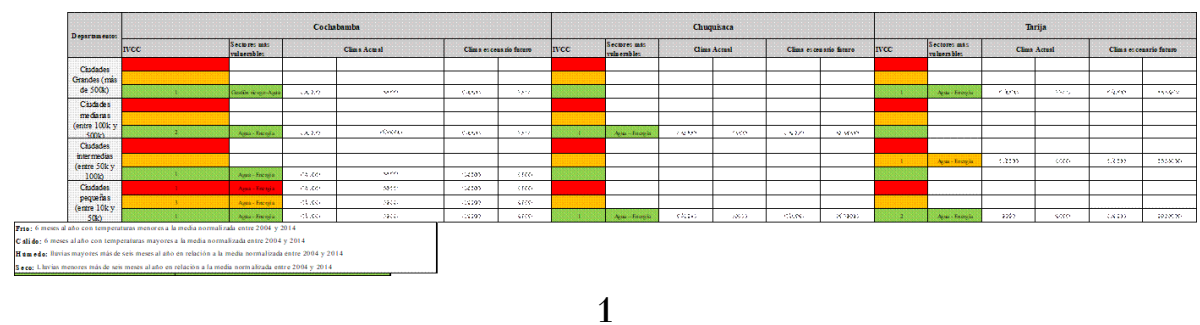

Caracterización de los municipios con centros urbanos de la región altiplánica - IVCC

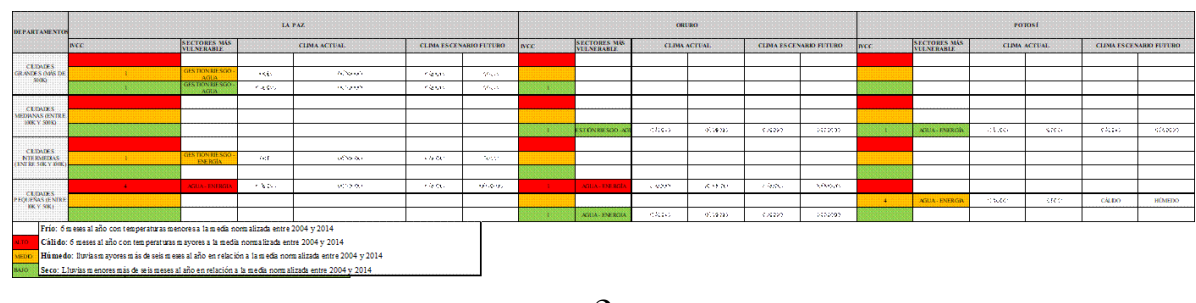

2

Caracterización de los municipios con centros urbanos de la región valles - IVCC Fuente: Elaboración Propia

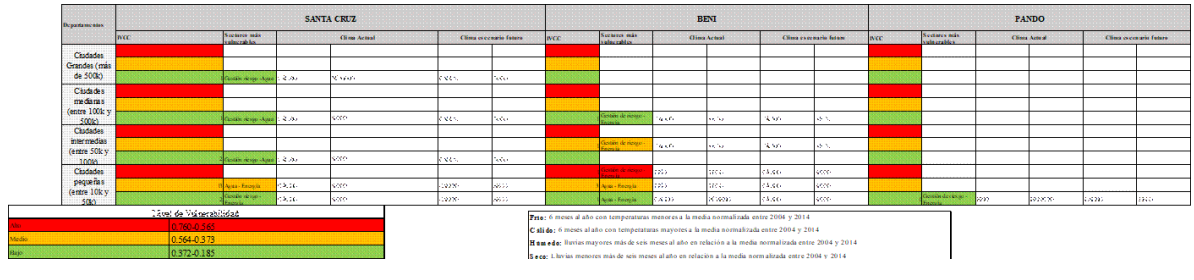

3

Caracterización de los municipios con centros urbanos de la región llanos y amazonas - IVCC

Fuente: Elaboración Propia 


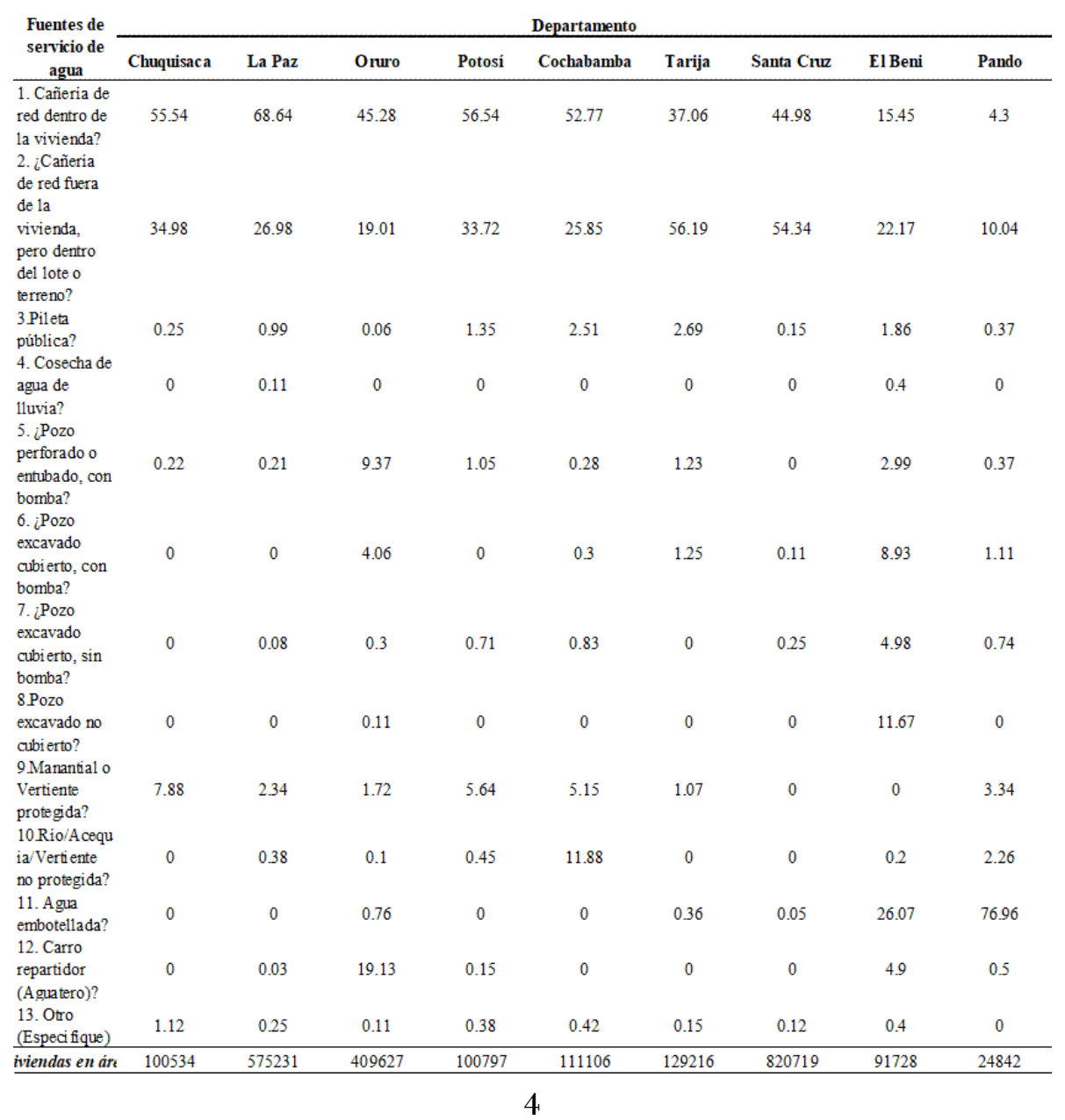

Tipo de fuente de agua por departamento en las zonas urbanas, Bolivia 2018 Fuente: elaboración propia con base en EH 2018

\section{Notas}

1 Según el Instituto Nacional de Estadística los municipios urbanos son aquellos con más de 500000 habitantes.

2 La investigación toma datos del modelo MIROC5 y 3, con una resolución de $\mathrm{X}=1.406^{\circ}, \mathrm{Y}=1.39^{\circ}$, generados por National Institute for Environmental Studies and Japan Agency for Marine-Earth Science and Technology, JAPÓN, y el Proyecto de Intercomparación de Modelos Acoplados, fase cinco (CMIP5) del IPCC, descargado del Centro Internacional para la Investigación del Fenómeno del Niño (CIIFEN) y del Servicio Nacional de Meteorología e Hidrología de Bolivia. Se seleccionó un escenario medio futuro (RCP 4.5) para predecir los efectos del cambio climático a mediano plazo. Este escenario medio tiene mayor probabilidad de observar años con características climáticas similares en la actualidad.

3 El índice tiene un valor de 0 a 1 , donde cero representa menor amenaza por cambio climático y uno es una mayor amenaza al cambio climático.

4 Es importante aclarar que la división se realiza con base en el valor mayor y menor que se generó con el índice, en este sentido no podemos afirmar que los municipios que presentan un índice menor de 0.33 no son vulnerables, sino que en comparación a los demás municipios se encuentran en una posición de menor vulnerabilidad.

5 Además, otros sectores de alta relevancia estratégica son el de salud y el de protección social, donde la capacidad de gestión es fundamental para poder reducir vulnerabilidades ante riesgos que puedan mermar el bienestar general.

6 Es importante aclarar que la división se realiza con base en el valor mayor y menor que se generó con el índice, en este sentido no podemos afirmar que los municipios que presentan un índice de 0.32 a 0.65 tienen una capacidad sectorial muy buena, sino que en comparación a los demás municipios se encuentran en una posición de mejor capacidad. 
S. Olivera-Villarroel, et al. La ruleta climática en Bolivia: Vulnerabilidad ante la incertidumbre...

7 Cabe recordar que en el artículo se considera capacidad institucional al gasto en mantenimiento y a la inversión que ejecutan las municipalidades en el sector.

8 En el anexo 2 se desagrega este indicador a nivel departamental de las zonas urbanas.

9 El programa Mi Agua se implementó en 2007, tuvo como objetivo financiar proyectos de agua potable en pequeñas comunidades del medio rural. El programa Mi Riego se implementó en 2008 este programa cuenta con 4 versiones y tiene el objetivo de financiar proyectos de riego en pequeñas comunidades del medio rural

10 En el anexo 1 se realiza una caracterización de los municipios según departamento, tamaño de la ciudad, sector vulnerable y cambio climático. 